Proceedings of the Edinburgh Mathematical Society (2008) 51, 305-313 (C)

DOI:10.1017/S0013091506001143 Printed in the United Kingdom

\title{
A CHARACTERIZATION OF COMPOSITION OPERATORS ON ALGEBRAS OF ANALYTIC FUNCTIONS
}

\author{
DANIEL CARANDO \\ Departamento de Matemática-Pab I, Facultad de Ciencias Exactas y Naturales, \\ Universidad de Buenos Aires, 1428 Buenos Aires, Argentina (dcarando@dm.uba.ar)
}

(Received 2 September 2006)

\begin{abstract}
We give a characterization of composition operators between algebras of analytic functions on a Banach space. We show (under fairly general conditions) that they are precisely the multiplicative operators that are transposes of operators of between the preduals of the algebras. The special cases of $H^{\infty}(U)$ and $H_{\mathrm{b}}(U)$ are considered. In these cases, the composition operators are those which are pointwise-to-pointwise continuous and/or $\tau_{0}$-to- $\tau_{0}$ continuous (where $\tau_{0}$ is the compact-open topology). We obtain Banach-Stone-type theorems for these algebras.
\end{abstract}

Keywords: composition operators; holomorphic functions; linearization of functions

2000 Mathematics subject classification: Primary 46G20; 46E50

Secondary 46J10

\section{Introduction}

In this paper we give a characterization of composition operators on algebras of analytic functions on a Banach space. Consider $\mathcal{F}(U)$, an algebra of scalar-valued analytic functions on an open subset $U$ of a Banach space $E$. An operator $A: \mathcal{F}(U) \rightarrow \mathcal{F}(V)$ is a composition operator if there exists a mapping $g: V \rightarrow U$ (of the vector-valued class $\mathcal{F}$, to be appropriately defined) such that $A f=f \circ g$ for each $f \in \mathcal{F}(U)$. Several authors have dealt with composition operators in different spaces or algebras of analytic functions on infinite dimensional spaces (see, for example, $[\mathbf{1}-\mathbf{3}, \mathbf{8}, \mathbf{1 2}]$ ). Many of these works relate properties of the operator $A$ (such as compactness, complete continuity, etc.) to properties of the function $g$ ('size' of the range, different kinds of continuity, etc.). Consequently, the representation of an operator $A$ as a composition operator proves useful for studying different characteristics of $A$. Motivated by this, we want to determine conditions for the existence of such a representation. In other words, we want a characterization of the operators $A: \mathcal{F}(U) \rightarrow \mathcal{F}(V)$, which are composition operators. For this, the existence of preduals of the algebra under consideration and the description of the multiplicative elements of these preduals are the main tools. We show under fairly general conditions that a multiplicative operator is a composition operator if and only if it is the transpose of a continuous operator between the preduals of the algebras. We also focus on two particular cases: the algebras of bounded holomorphic functions $H^{\infty}(U)$ and of holomorphic 
functions of bounded type $H_{\mathrm{b}}(U)$. In both cases, we give more equivalent conditions for a mapping to be a composition operator. This allows us to prove a Banach-Stone-type result for these algebras.

In the next section we describe a general construction linearizing function. This construction is taken from [4] and we briefly sketch it for completeness and to fix notation. Section 3 deals with the general results. We consider $\mathcal{F}(U)$, an algebra of analytic functions on $U$ where polynomials are weak-* dense $(U$ is an $\mathcal{F}$-regular open subset of a Banach space with the bounded approximation property). We show that a multiplicative operator $A: \mathcal{F}(U) \rightarrow \mathcal{F}(V)$ is a composition operator if and only if $A=T^{\prime}$, where $T$ is a continuous linear operator between the preduals of $\mathcal{F}(V)$ and $\mathcal{F}(U)$. In $\S 4$ we restrict ourselves to the algebras of bounded holomorphic functions and holomorphic functions of bounded type. We show that in these cases being a composition operator is equivalent to being pointwise-to-pointwise continuous and also being $\tau_{0}$-to- $\tau_{0}$ continuous. Finally, we find, under certain conditions, that if the algebras $\left(H^{\infty}(U), \tau_{0}\right)$ and $\left(H^{\infty}(V), \tau_{0}\right)$ are isomorphic, the Banach spaces containing $U$ and $V$ must be linearly isomorphic. An analogous result holds for the algebra $H_{\mathrm{b}}(U)$.

\section{Preliminaries}

In [4], a general construction linearizing functions with values in locally convex spaces is given. We briefly describe this construction.

First, we take a space $\mathcal{F}(U)$ of functions $f: U \rightarrow \mathbb{C}$ which are continuous. A space $\mathcal{F}_{*}(U)$ and a map $e: U \rightarrow \mathcal{F}_{*}(U)$ are constructed such that, for any $f \in \mathcal{F}(U)$, there exists an $L_{f} \in \mathcal{F}_{*}(U)^{\prime}$ such that $L_{f} \circ e=f$. A sketch of this construction follows.

Consider the vector space $\mathbb{C}^{(U)}$ of finitely supported families of $U$-indexed complex numbers. A typical element will be denoted by $s=\sum_{x \in U} a_{x} e_{x}$, with $e_{x}(y)=\delta_{x y}$. Note that the sum is finite. For any given $f \in \mathcal{F}(U)$, we define the seminorm

$$
p_{f}(s)=\left|\sum_{x \in U} a_{x} f(x)\right| .
$$

Now

$$
\mathcal{N}=\left\{s \in \mathbb{C}^{(U)}: \sum_{x \in U} a_{x} f(x)=0 \text { for all } f \in \mathcal{F}(U)\right\}
$$

is a subspace of $\mathbb{C}^{(U)}$, and we define $X$ as the quotient

$$
X=\mathbb{C}^{(U)} / \mathcal{N} .
$$

We will continue to denote the class of $e_{x}$ by $e_{x}$, and the class of $s$ by $s=\sum_{x \in U} a_{x} e_{x}$. We endow $X$ (just for a moment) with the topology $\tau$ generated by the seminorms $p_{f}$. It is clear that a function $f \in \mathcal{F}(U)$ factors through $e$ in the following way:

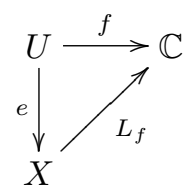


where $L_{f}(s)=\sum_{x} a_{x} f(x)$ if $s=\sum_{x} a_{x} e_{x}$. The map $\mathcal{F}(U) \rightarrow X^{\prime}$ defined by $f \mapsto L_{f}$ is then an algebraic isomorphism.

Next, we consider on $X$ the strongest locally convex topology compatible with $\tau$ for which the map $e: U \rightarrow X$ is continuous, and denote $X$ with this topology by $(X, \alpha)$. We take $\mathcal{F}_{*}(U)$ to be the completion of $(X, \alpha)$ :

$$
\mathcal{F}_{*}(U)=\widehat{(X, \alpha)} .
$$

We still denote by $e$ the continuous map $U \rightarrow X \rightarrow \mathcal{F}_{*}(U)$. In fact, the topology $\alpha$ on $\mathcal{F}(U)_{\alpha}^{\prime}$ is the topology of uniform convergence on the equicontinuous $\tau_{p^{-}}$-compact discs of $\mathcal{F}(U)$ ( $\tau_{p}$ denotes the topology of pointwise convergence). Here, equicontinuous means equicontinuous as functions on $U$.

We can identify $\mathcal{F}(U)$ with $\mathcal{F}_{*}(U)^{\prime}$. Moreover, in many situations $\mathcal{F}(U)$ is the strong dual of $\mathcal{F}_{*}(U)$. This happens, for example, with $H_{\mathrm{b}}(U)$ or $H^{\infty}(U)$. Although there may be more than one topology of interest on $\mathcal{F}(U)$, we will consider on $\mathcal{F}(U)$ the topology which makes it the strong dual of $\mathcal{F}_{*}(U)$.

Given a class $\mathcal{F}(U)$ of continuous scalar-valued functions and a locally convex space $F$, we will say that $g: U \rightarrow F$ is weakly in $\mathcal{F}$ if $g$ is continuous and, for every $\gamma \in F^{\prime}$, $\gamma \circ g \in \mathcal{F}(U)$. We denote by $\omega \mathcal{F}(U, F)$ the space of all such functions. For bounded holomorphic functions or holomorphic functions of bounded type, this coincides with the vector-valued notion of these classes of functions (see $\S 4$ for the definitions). $\mathcal{F}_{*}(U)$ also factors functions on $\omega \mathcal{F}(U, F)$, as follows.

Theorem 2.1 (Carando and Zalduendo [4, Theorem 3]). Each function in $\omega \mathcal{F}(U, F)$ factors linearly through $e$ :

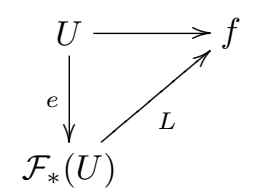

identifying $L\left(\mathcal{F}_{*}(U), F\right)$ with $\omega \mathcal{F}(U, F)$ algebraically.

\section{General results}

Let $U$ be an open subset of the a locally convex space $E$. If the class $\mathcal{F}(U)$ contains $E^{\prime}$, the mapping $e$ defined in the previous section is one-to-one [4, Proposition 1]. Moreover, if $E$ is a Banach space, $e$ is in fact bicontinuous.

Lemma 3.1. If $E$ is a Banach space and $\mathcal{F}(U)$ contains $E^{\prime}$, then $U$ is homeomorphic to $e(U)$.

Proof. Since $e$ is always continuous, we need only show that the topology $\alpha$ on $e(U)$ is stronger than that induced by the norm. This is a consequence of the fact that the unit ball of $E^{\prime}$ is one of the equicontinuous $\tau_{p}$-compact discs in $\mathcal{F}(U)$ which define the topology $\alpha$. 
If $E^{\prime} \subset \mathcal{F}(U)$, we can consider the projection $\pi: \mathcal{F}(U)^{\prime} \rightarrow E^{\prime \prime}$ given by $\pi(\phi)=\left.\phi\right|_{E^{\prime}}$. When dealing with some algebras of analytic functions, evaluation on elements of the bidual $E^{\prime \prime}$ is defined, so the range of $\pi$ contains elements in $E^{\prime \prime}$. However, the elements of $\mathcal{F}_{*}(U)$ are always mapped into $E$, i.e. $\pi\left(\mathcal{F}_{*}(U)\right)$ is contained in $E$.

Proposition 3.2. Let $E$ be a Banach space and suppose that $E^{\prime} \subset \mathcal{F}(U)$. If $\phi \in$ $\mathcal{F}_{*}(U)$, then $\pi(\phi)$ belongs to $E$.

Proof. Let $z=\pi(\phi)$ and take $\left(s_{t}\right)_{t} \subset X$ such that $s_{t} \rightarrow \phi$ in the topology $\alpha$. Each $s_{t}$ can be written as

$$
s_{t}=\sum a_{i}^{t} e_{x_{i}^{t}} .
$$

Let $x_{t}=\sum a_{i}^{t} x_{i}^{t}$. For $\gamma \in B_{E^{\prime}}$ we have that

$$
\gamma\left(x_{t}\right)=\sum a_{i}^{t} \gamma\left(x_{i}^{t}\right)=L_{\gamma}\left(s_{t}\right)
$$

which converges to $L \gamma(\phi)=z(\gamma)$ uniformly for $\gamma \in B_{E^{\prime}}$. This means that $x_{t}$ converges to $z$ in the norm and then $z \in E$.

We now assume that $\mathcal{F}(E)$ is an algebra of analytic functions and denote by $\mathcal{F}_{*}^{M}(E)$ the set of elements in $\mathcal{F}_{*}(E)$ that are multiplicative. Clearly, $e(E)$ is contained in $\mathcal{F}_{*}(E)$. We see that these two sets often coincide.

Proposition 3.3. Let $E$ be a Banach space with the bounded approximation property and let $\mathcal{F}(E)$ be an algebra of analytic functions where polynomials are $\sigma\left(\mathcal{F}(E), \mathcal{F}_{*}(E)\right.$ )dense. Then $\mathcal{F}_{*}^{M}(E)=e(E)$.

Proof. Let $\Phi \in \mathcal{F}_{*}^{M}(E)$ and let $z=\left.\Phi\right|_{E^{\prime}}$. Proposition 3.2 implies that $z \in E$. Let us see that $\Phi(P)=P(z)$ for every $P \in P\left({ }^{k} E\right)$, for any $k \in \mathbb{N}$.

We fix $P$. By the bounded approximation property, for every compact subset $K \subset E$ and every $\varepsilon>0$ there exists a finite-type polynomial $P_{K, \varepsilon}$ such that $\left\|P-P_{K, \varepsilon}\right\|_{K}<\varepsilon$ and the collection $\left(P_{K, \varepsilon}\right)_{K, \varepsilon}$ is bounded. Note that, since $P_{K, \varepsilon}$ is a finite-type polynomial and $\Phi$ is linear and multiplicative,

$$
\Phi\left(P_{K, \varepsilon}\right)=P_{K, \varepsilon}(z) .
$$

Let $\left(s_{t}\right)_{t} \subset X$ such that $s_{t} \stackrel{\alpha}{\rightarrow} \Phi$. If we define $B=\Gamma \overline{\left\{P_{K, \varepsilon}\right\} \cup\{P\}} \subset P\left({ }^{k} E\right)$, then $s_{t} \rightarrow$ $\Phi$ uniformly on $B$ ( $B$ is bounded and therefore equicontinuous and $\tau_{p}$-compact). Given $\varepsilon>0$, there exists $t_{0}$ such that

$$
\left|L_{Q}\left(s_{t_{0}}\right)-\Phi(Q)\right|<\varepsilon \quad \forall Q \in B
$$

Since $s_{t_{0}}$ can be written as $s_{t_{0}}=\sum a_{i} x_{i}$ (finite sum), there exists a finite-type polynomial $P_{0} \in B$ such that

$$
\begin{aligned}
\left|L_{P_{0}}\left(s_{t_{0}}\right)-L_{P}\left(s_{t_{0}}\right)\right| & <\varepsilon \\
\left|L_{P_{0}}(z)-L_{P}(z)\right| & \left.<\varepsilon \quad \text { (equivalently, }\left|P_{0}(z)-P(z)\right|<\varepsilon\right) .
\end{aligned}
$$


So we have

$$
\begin{aligned}
|\Phi(P)-P(z)| \leqslant & \left|\Phi(P)-L_{P}\left(s_{i_{0}}\right)\right|+\left|L_{P}\left(s_{i_{0}}\right)-L_{P_{0}}\left(s_{i_{0}}\right)\right| \\
& \quad+\left|L_{P_{0}}\left(s_{i_{0}}\right)-\Phi\left(P_{0}\right)\right|+\left|\Phi\left(P_{0}\right)-P_{0}(z)\right|+\left|P_{0}(z)-P(z)\right| \\
<\varepsilon & +\varepsilon+\varepsilon+0+\varepsilon
\end{aligned}
$$

by $(3.1)-(3.4)$.

Consequently, $\Phi(P)=P(z)$ for every $P \in P\left({ }^{k} E\right)$, for any $k \in \mathbb{N}$, and, by density, $\Phi=e_{z}$.

Let $U$ be an open subset of the Banach space $E$ and $\mathcal{F}(U)$ be an algebra of analytic functions in which polynomials are $\sigma\left(\mathcal{F}(U), \mathcal{F}_{*}(U)\right)$-dense. We define

$$
U_{\mathcal{F}}:=\pi\left(\mathcal{F}_{*}^{M}(U)\right) .
$$

Given $\Phi$ in $\mathcal{F}_{*}^{M}(U)$ we set $z=\pi(\Phi)$. As in the previous proposition, we can see that $\Phi(P)=P(z)$ for each polynomial $P$ on $E$. As a consequence, if $\pi\left(\Phi_{1}\right)=\pi\left(\Phi_{2}\right)$, we obtain $\Phi_{1}(P)=\Phi_{2}(P)$ for all $P$ and, by density, $\Phi_{1}=\Phi_{2}$. Therefore, we have that $\pi$ is a bijection between $U_{\mathcal{F}}$ and $\mathcal{F}_{*}^{M}(U)$.

If $f \in \mathcal{F}(U)$, we can define $f_{\mathcal{F}}: U_{\mathcal{F}} \rightarrow \mathbb{C}$ by $f_{\mathcal{F}}(z)=\Phi(f)$, where $\pi(\Phi)=z$. The remarks in the previous paragraph show that $f_{\mathcal{F}}$ is well defined. Note that the mapping $f \mapsto f_{\mathcal{F}}$ is linear and multiplicative. Moreover, if we take $z \in U_{\mathcal{F}}$ and define $e^{\mathcal{F}}(z)$ by $e^{\mathcal{F}}(z)(f)=f_{\mathcal{F}}(z)$, we have a mapping $e^{\mathcal{F}}: U_{\mathcal{F}} \rightarrow \mathcal{F}_{*}^{M}(U)$, which is the inverse of $\pi$. Also, $e^{\mathcal{F}}$ is an extension of the mapping $e$ defined in the previous section. With this notation we have the following lemma.

Lemma 3.4. Let $E$ be a Banach space with the bounded approximation property. Let $\mathcal{F}(U)$ be an algebra of analytic functions where polynomials are $\sigma\left(\mathcal{F}(U), \mathcal{F}_{*}(U)\right)$-dense. Then $\mathcal{F}_{*}^{M}(U)=e^{\mathcal{F}}\left(U_{\mathcal{F}}\right)$.

We will say that an open subset $U$ of $E$ is $\mathcal{F}$-regular if $U_{\mathcal{F}}=U$. Clearly, if $U$ is $\mathcal{F}$ regular, $e$ coincides with $e^{\mathcal{F}}$. In what follows, $V$ will be an open subset of a Banach space $G$. When necessary, we will use the notation $e_{U}$ for the mapping $e$ associated with an open set $U$.

In this setting, a mapping $A: \mathcal{F}(U) \rightarrow \mathcal{F}(V)$ will be called a composition operator if there exists a $g \in w \mathcal{F}(V, U)$ such that $A f=f \circ g$ for all $f \in \mathcal{F}(U)$. We have the following characterization of composition operators.

Theorem 3.5. Let $E$ be a Banach space with the bounded approximation property and let $\mathcal{F}(U)$ be an algebra of analytic functions on $U$ where polynomials are $\sigma\left(\mathcal{F}(U), \mathcal{F}_{*}(U)\right)$-dense. Suppose that $U$ is $\mathcal{F}$-regular and let $A: \mathcal{F}(U) \rightarrow \mathcal{F}(V)$ be a homomorphism. Then $A$ is a composition operator if and only if $A=T^{\prime}$, where $T: \mathcal{F}_{*}(V) \rightarrow \mathcal{F}_{*}(U)$ is a continuous operator.

Proof. If $A: \mathcal{F}(U) \rightarrow \mathcal{F}(V)$ is a composition operator, say $A(f)=f \circ g$, we can consider the mapping $e_{U} \circ g: V \rightarrow \mathcal{F}_{*}(U)$. For each $L=L_{f} \in\left(\mathcal{F}_{*}(U)\right)^{\prime}$, we have

$$
L_{f} \circ e_{U} \circ g=f \circ g=A(f) \in \mathcal{F}(V) .
$$


Therefore, $e_{U} \circ g$ belongs to $w \mathcal{F}\left(V, \mathcal{F}_{*}(U)\right)$ and there exists a continuous operator

$$
T=T_{e_{E} \circ g}: \mathcal{F}_{*}(V) \rightarrow \mathcal{F}_{*}(U)
$$

such that $e_{U} \circ g=T \circ e_{V}$. It is easy to check that $A=T^{\prime}$.

Conversely, suppose $A=T^{\prime}$. Since $A$ is a multiplicative operator, $T(e(V))$ is contained in $\mathcal{F}_{*}^{M}(U)=e(U)$ (the latter equality follows from the previous lemma and the fact that $U$ is $\mathcal{F}$-regular). Therefore, we can define $g: V \rightarrow U$ by $g(y)=x$ if $T\left(e_{y}\right)=e_{x}$. Now $A f(y)=T^{\prime}\left(L_{f}\right)\left(e_{y}\right)=L_{f}\left(T\left(e_{y}\right)\right)=L_{f}\left(e_{g(y)}\right)=f(g(y))$ and it follows that $A f=f \circ g$.

Let us see that $g$ belongs to $w \mathcal{F}(V, U)$. By Lemma 3.1, $U$ and $V$ are homeomorphic to $e(U)$ and $e(V)$ and, since $T$ is continuous, $g$ is continuous. Moreover, if $\gamma \in E^{\prime}$, $\gamma \circ g=A \gamma \in \mathcal{F}(V)$. Thus, $g \in w \mathcal{F}(V, U)$.

Suppose that $U$ is not $\mathcal{F}$-regular but that there exists a $g \in w \mathcal{F}(V, E)$ whose image is contained in $U_{\mathcal{F}}$ such that $A f(x)=f_{\tau} \circ g(x)$ for all $x \in V$. We can then find $T: \mathcal{F}_{*}(V) \rightarrow \mathcal{F}_{*}(V)$ such that $A=T^{\prime}$ just as before. Conversely, if $A=T^{\prime}$, the previous construction leads us to a mapping $g: V \rightarrow U_{\mathcal{F}}$ such that $A f(x)=f_{\tau} \circ g(x)$. In fact, $g$ belongs to $w \mathcal{F}(V, E)$. To see this, we must show that $g$ is continuous. But

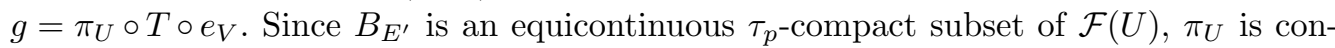
tinuous from $\left(\mathcal{F}_{*}^{M}(U), \alpha\right)$ to $\left(U_{\mathcal{F}},\|\cdot\|\right)$ and therefore so is $g$. Also, $\gamma \circ g=A(\gamma) \in \mathcal{F}(U)$ for all $\gamma \in E^{\prime}$. We have shown the following result.

Corollary 3.6. Let $E$ be a Banach space with the bounded approximation property and let $\mathcal{F}(U)$ be an algebra of analytic functions on $U$ where polynomials are $\sigma\left(\mathcal{F}(U), \mathcal{F}_{*}(U)\right.$ )-dense. Let $A: \mathcal{F}(U) \rightarrow \mathcal{F}(V)$ be a homomorphism. The following are equivalent:

(a) there exists a function $g \in w \mathcal{F}(V, E)$ whose image is contained in $U_{\mathcal{F}}$ such that $A f(x)=f_{\mathcal{F}} \circ g(x)$ for all $x \in V$;

(b) $A=T^{\prime}$, for some continuous operator $T: \mathcal{F}_{*}(V) \rightarrow \mathcal{F}_{*}(U)$.

In the previous results, the hypotheses were used only in one direction. With the same proof, we see that a composition operator is the transpose of an operator between the preduals in a much more general situation, as follows.

Proposition 3.7. Let $E$ and $G$ be locally convex spaces and let $U \subset E$ and $V \subset G$ be open subsets. If $A: \mathcal{F}(U) \rightarrow \mathcal{F}(V)$ is a composition operator, then there exists a continuous operator $T: \mathcal{F}_{*}(V) \rightarrow \mathcal{F}_{*}(U)$ such that $A=T^{\prime}$.

\section{Operators on $H^{\infty}(U)$ and $H_{\mathrm{b}}(U)$}

We denote by $H^{\infty}(U)$ the space of bounded holomorphic functions on $U$. This is a Banach space when equipped with the supremum norm. Mujica [10] has constructed a Banach predual $G_{\infty}(U)$ of $H^{\infty}(U)$. A characterization of this predual is

$$
G_{\infty}(U)=\left\{\phi \in H^{\infty}(U)^{\prime}: \phi \text { is } \tau_{0} \text {-continuous on the unit ball of } H^{\infty}(U)\right\} .
$$


The construction given in $\S 2$ coincides with $G_{\infty}(U)[4]$. We obtain the following characterization of composition operators on $H^{\infty}(U)$.

Theorem 4.1. Let $A: H^{\infty}(U) \rightarrow H^{\infty}(V)$ be a multiplicative operator. If $E$ has the bounded approximation property and $U$ is bounded and absolutely convex, then the following conditions are equivalent:

(a) $A$ is a composition operator;

(b) $A=T^{\prime}$ for some continuous operator $T: G_{\infty}(V) \rightarrow G_{\infty}(U)$;

(c) $A$ is $\tau_{p}$-to- $\tau_{p}$ continuous;

(d) $A$ is $\tau_{0}-$ to- $\tau_{0}$ continuous.

Proof. (a) $\Longrightarrow$ (c) and (a) $\Longrightarrow$ (d) are straightforward. We suppose that (c) holds and let $\phi \in G_{\infty}(V)$. By (c) and the characterization of $G_{\infty}(V), \phi \circ A$ is $\tau_{p}$-continuous on the unit ball of $H^{\infty}(U)$. The topologies $\tau_{p}$ and $\tau_{0}$ coincide on this unit ball $[6$, Lemma 3.25]. We then have that $\phi \circ A$ is $\tau_{0}$-continuous on the unit ball of $H^{\infty}(U)$ and consequently $\phi \circ A$ belongs to $G_{\infty}(U)$. This shows that $A^{\prime}: H^{\infty}(V)^{\prime} \rightarrow H^{\infty}(U)^{\prime}$ maps $G_{\infty}(V)$ in $G_{\infty}(U)$. Therefore, $A$ is the transpose of the restriction of $A^{\prime}$ to $G_{\infty}(V)$ and we have (b). In an analogous way we have (d) $\Longrightarrow(\mathrm{b})$.

Finally, to see that (b) $\Longrightarrow$ (a) let us check that we may apply Theorem 3.5. Since $U$ is absolutely convex, it is $H^{\infty}$-regular. Indeed, if $z \in U_{H^{\infty}} \backslash U$, we choose $\gamma \in E^{\prime}$ such that $\gamma(z)=1$ and $\sup _{x \in U}|\gamma(x)|<1$. The sequence $\left(\gamma^{n}\right)_{n}$ is in the unit ball of $H^{\infty}(U)$ and $\tau_{0}$-converges to zero. If $\Phi$ is such that $\pi(\Phi)=z$, we have on the one hand that $\Phi\left(\gamma^{n}\right) \rightarrow 0$. On the other hand, $\Phi\left(\gamma^{n}\right)=\Phi(\gamma)^{n}=\gamma(z)^{n}=1$, which is a contradiction. Moreover, polynomials are $\left(H^{\infty}(U), G_{\infty}(U)\right)$-dense: the Taylor series expansion of a function $f \in$ $H^{\infty}(U) \tau_{0}$-converges to $f$ and is bounded. Since elements in $G_{\infty}(U)$ are $\tau_{0}$-continuous on bounded subsets of $H^{\infty}(U)$, density follows. Now (a) is a consequence of Theorem 3.5.

Now we turn our attention to holomorphic functions of bounded type. We say that $B \subset U$ is a $U$-bounded set if it is bounded and $\operatorname{dist}(B, E \backslash U)>0$. We will denote by $H_{\mathrm{b}}(U)$ the space of holomorphic functions $f: U \rightarrow \mathbb{C}$ that are bounded on $U$-bounded sets, i.e. $\|f\|_{B}:=\sup \{|f(x)|: x \in B\}<\infty$ for all $U$-bounded sets $B . H_{\mathrm{b}}(U)$ is a Fréchet algebra when endowed with the topology of the uniform convergence on $U$-bounded subsets of $U$. A fundamental sequence of $U$-bounded sets is given by

$$
U_{n}=\left\{x \in U:\|x\|<n \text { and } d\left(x, U^{\mathrm{c}}\right)>\frac{1}{n}\right\} .
$$

The topology on $H_{\mathrm{b}}(U)$ is given by uniform convergence on each $U_{n}$.

When the construction described in $\S 2$ is applied to $H_{\mathrm{b}}(U)$, we obtain the space $\mathcal{P}_{\mathrm{b}}(U)$, constructed in [7]. A characterization of this space was given in [11]: for each sequence of positive numbers $\left(\alpha_{n}\right)_{n}$, we define

$$
B^{\alpha}=\left\{f \in H_{\mathrm{b}}(U): \sup _{U_{n}}|f| \leqslant \alpha_{n}\right\}
$$


Now, $\mathcal{P}_{\mathrm{b}}(U)$ is the set of all $\phi \in H_{\mathrm{b}}(U)^{\prime}$ that are $\tau_{0}$-continuous on $B^{\alpha}$ for all $\alpha$. In [7, Proposition 3], the authors give a description of the multiplicative elements of $\mathcal{P}_{\mathrm{b}}(E)$ when $E$ has the approximation property.

If $U \subset E$ is open and absolutely convex, using the proof that showed the $H^{\infty}$-regularity of $U$, we can see that it is $H_{\mathrm{b}}$-regular. Also, polynomials are $\left(H_{\mathrm{b}}(U), \mathcal{P}_{\mathrm{b}}(U)\right)$-dense. In fact, they are dense with the strong dual topology on $H_{\mathrm{b}}(U)$. Indeed, the Taylor series expansion of a function $f \in H_{\mathrm{b}}(U)$ converges uniformly on $U$-bounded sets. But uniform convergence on $U$-bounded sets is precisely the strong dual topology on $H_{\mathrm{b}}(U)$. We have the following result.

Theorem 4.2. Let $E$ be a Banach space with the bounded approximation property and let $U \subset E$ and $V \subset G$ be open subsets, with $U$ absolutely convex. If $A: H_{\mathrm{b}}(U) \rightarrow$ $H_{\mathrm{b}}(V)$ is a homomorphism, the following are equivalent:

(a) $A$ is a composition operator;

(b) $A=T^{\prime}$, where $T: \mathcal{P}_{\mathrm{b}}(V) \rightarrow \mathcal{P}_{\mathrm{b}}(U)$ is a continuous operator;

(c) $A$ is $\tau_{p}$-to- $\tau_{p}$ continuous;

(d) $A$ is $\tau_{0}$-to- $\tau_{0}$ continuous.

Proof. Again, (a) $\Longrightarrow$ (c) and (a) $\Longrightarrow$ (d) are straightforward. We suppose that (c) holds and let $\phi \in \mathcal{P}_{\mathrm{b}}(V)$. By (c) and the characterization of $\mathcal{P}_{\mathrm{b}}(V), \phi \circ A$ is $\tau_{p}$-continuous on each $B^{\alpha}$. Since the subsets $B_{\alpha}$ are locally bounded, the topologies $\tau_{p}$ and $\tau_{0}$ coincide on each $B^{\alpha}$ [6, Lemma 3.25]. We have that $\phi \circ A$ is $\tau_{0}$-continuous on each $B^{\alpha}$ and consequently $\phi \circ A$ belongs to $\mathcal{P}_{\mathrm{b}}(U)$. This shows that $A^{\prime}: H(V)^{\prime} \rightarrow H(U)^{\prime}$ maps $\mathcal{P}_{\mathrm{b}}(V)$ in $\mathcal{P}_{\mathrm{b}}(U)$. Therefore, $A$ is the transpose of the restriction of $A^{\prime}$ to $\mathcal{P}_{\mathrm{b}}(V)$ and we have (b). In an analogous way we have (d) $\Longrightarrow(b)$. (b) $\Longrightarrow$ (a) follows from Theorem 3.5.

If both $U$ and $V$ satisfy the conditions of Theorem 4.1 and the operator $A$ is an isomorphism, the function $g$ is invertible, with $g^{-1}$ in $H^{\infty}(U, V)$. Therefore, an isomorphism between the algebras $\left(H^{\infty}(U), \tau_{0}\right)$ and $\left(H^{\infty}(U), \tau_{0}\right)$ is equivalent to the analytic equivalence of $U$ and $V$. The existence of such biholomorphic functions implies that the spaces $E$ and $G$ are isomorphic as Banach spaces, since the differential at any point is an isomorphism. Moreover, if $U$ and $V$ are the unit balls of $E$ and $G$, the isomorphism is isometric [9]. The situation for $H_{\mathrm{b}}(U)$ is analogous. Thus, we have the following results (see also $[\mathbf{5}, \mathbf{1 3}]$, where similar results have been obtained for different algebras).

Corollary 4.3. Let $U \subset E$ and $V \subset G$ be bounded, open and absolutely convex and suppose that $E$ and $G$ have the bounded approximation property. Then $\left(H^{\infty}(U), \tau_{0}\right)$ and $\left(H^{\infty}(V), \tau_{0}\right)$ are topological and algebraically isomorphic if and only if $U$ and $V$ are holomorphically equivalent. In this case, $E$ and $G$ are isomorphic Banach spaces. If $U$ and $V$ are the unit balls of $E$ and $G$, the spaces are isometric. 
Corollary 4.4. Let $U \subset E$ and $V \subset G$ open and absolutely convex and suppose that $E$ and $G$ have the bounded approximation property. Then $\left(H_{\mathrm{b}}(U), \tau_{0}\right)$ and $\left(H_{\mathrm{b}}(V), \tau_{0}\right)$ are topological and algebraically isomorphic if and only if $U$ and $V$ are holomorphically equivalent. In this case, $E$ and $G$ are isomorphic Banach spaces. If $U$ and $V$ are the unit balls of $E$ and $G$, the spaces are isometric.

Acknowledgements. This work was partly supported by PIP 5272, UBACyT X108 and PICT 03-15033. The author thanks Chris Boyd, Manolo Maestre and Nacho Zalduendo for very helpful conversations and suggestions.

\section{References}

1. R. Aron, P. Galindo And M. Lindström, Compact homomorphisms between algebras of analytic functions, Studia Math. 123 (1997), 235-247.

2. J. BONET AND M. FRIZ, Weakly compact composition operators on locally convex spaces, Math. Nachr. 245 (2002), 26-44.

3. J. Bonet, P. Domański, M. Lindström and J. Taskinen, Composition operators between wighted Banach spaces of analytic functions, J. Austral. Math. Soc. A 64 (1998), 101-118.

4. D. Carando and I. Zalduendo, Linearization of functions, Math. Ann. 328 (2004), 683-700.

5. D. Carando, D. García and M. Maestre, Homomorphisms and composition operators on algebras of analytic functions of bounded type, Adv. Math. 197 (2005), 607-629.

6. S. Dineen, Complex analysis on infinite dimensional spaces, Springer Monographs in Mathematics, Volume 198 (Springer, 1999).

7. P. Galindo, D. García and M. Maestre, Holomorphic mappings of bounded type, $J$. Math. Analysis Applic. 166 (1992), 236-246.

8. D. García, M. Maestre and P. Sevilla, Composition operators on weighted spaces of holomorphic functions on Banach spaces, Ann. Acad. Sci. Fenn. Math. 29 (2004), 81-98.

9. W. KAUP AND H. UPMEIER, Banach spaces with biholomorphically equivalent unit balls are isomorphic, Proc. Am. Math. Soc. 58 (1976), 129-133.

10. J. MuJICA, Linearization of bounded holomorphic mappings on Banach spaces, Trans. Am. Math. Soc. 324 (1991), 867-887.

11. J. MuJICA, Linearization of holomorphic mappings of bounded type, in Progress in functional analysis, pp. 149-162 (North-Holland, Amsterdam, 1992).

12. J. TASKINEn, Compact composition operators on general weighted spaces, Houston J. Math. 27 (2001), 203-218.

13. D. VIEIRA, Theorems of Banach-Stone type for algebras of holomorphic functions on infinite dimensional spaces, Math. Proc. R. Irish Acad. A 106 (2006), 97-113. 